K. Sato

Nagoya Math. J.

Vol. $104(1986), 29-42$

\title{
BOUNDS OF MODES AND UNIMODAL PROCESSES WITH INDEPENDENT INCREMENTS
}

\author{
KEN-ITI SATO
}

\section{$\S 1$. Introduction}

A probability measure $\mu$ is called unimodal if there is a point $a$ such that the distribution function of $\mu$ is convex on $(-\infty, a)$ and concave on $(a, \infty)$. The point $a$ is called a mode of $\mu$. When $\mu$ is unimodal, the mode of $\mu$ is not always unique; the set of modes is a one point set or a closed interval. If $\mu$ is a unimodal distribution with finite variance, Johnson and Rogers [6] give a bound

$$
|a-m| \leq \sqrt{3 v}
$$

where $m$ and $v$ are mean and variance of $\mu$ (see also [11]). Here $\sqrt{3}$ is the best constant. Let $\beta_{p}$ be the absolute moment (possibly infinite) of $\mu$ of order $p$. We will extend the method of [6] and give a bound

$$
|a| \leq \operatorname{const} \beta_{p}^{1 / p}
$$

for any (not necessarily integer) $p>0$. The constant depends only on $p$. We can give it explicitly, although it is not the best constant. Inequalities of the type (1.2) are proved in Section 2. We emphasize that they apply to distributions for which $\beta_{p}$ is finite only for small $p$, such as nonGaussian stable distributions.

In Section 3 we consider a stochastic process $X_{t}$ with homogeneous independent increments. Some behaviors of its absolute moments as $t \rightarrow \infty$ are given. We use them to show some limit theorems of modes when $X_{t}$ is unimodal. The inequalities in Section 2 can be used to give explicit bounds in the behaviors of modes. In Section 4 the modes of stable processes with index 1 are examined.

Bounds of modes for special classes of unimodal distributions are treated in some papers. Wolfe [12] and Sato-Yamazato [9] consider distri-

Received August 9, 1984. 
butions of class $L$. Hall [4] studies unimodal sums of i.i.d. random variables in the domain of attraction of the Gaussian distribution.

\section{$\S 2$. Bound of modes}

The following lemma is basic to our discussion. It is suggested by a discussion in Johnson and Rogers [6].

Lemma 2.1. If $\mu$ is unimodal and the origin is a mode, then

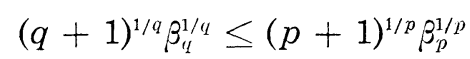

for any $p>q>0$.

Proof. By a result of Khintchine and Shepp ([1] V.9), the distribution of $X$ is unimodal with a mode 0 if and only if there are independent random variables $U$ and $Y$ such that $U$ is uniformly distributed on $[0,1]$ and $U Y$ has the identical distribution with $X$. Hence $\beta_{p}=E|X|^{p}=$ $E U^{p} E|Y|^{p}=(p+1)^{-1} E|Y|^{p}$, if $X$ has distribution $\mu$. Now (2.1) is a consequence of the moment inequality ([1] V.8) applied to the absolute moments of $Y$.

TheOREm 2.1. Let $\mu$ be a unimodal probability measure. Let a be a mode of $\mu$. Then,

$$
\left(q+1-(p+1)^{q / p}\right)|a|^{q} \leq(q+1) \beta_{q}+(p+1)^{q / p} \beta_{p}^{q / p}
$$

for any $p$ and $q$ satisfying $0<q \leq 1$ and $q<p$.

Note that $(x+1)^{1 / x}$ is decreasing in $x>0$, so the coefficient of $|a|^{q}$ is positive.

Proof. Let $X$ be a random variable with distribution $\mu$. Then $X-a$ is unimodal with a mode 0. Applying Lemma 2.1, we have

$$
(q+1)^{1 / q}\left(E|X-a|^{q}\right)^{1 / q} \leq(p+1)^{1 / p}\left(E|X-a|^{p}\right)^{1 / p}
$$

for $0<q<p$. We use $\left.|| x\right|^{\alpha}-|y|^{\alpha}|\leq| x-\left.y\right|^{\alpha} \leq|x|^{\alpha}+|y|^{\alpha}$ for $0<\alpha \leq 1$. If $0<q<p \leq 1$, then it follows that

$$
\begin{aligned}
\left.(q+1)|| a\right|^{q}-\beta_{q} \mid & \leq(p+1)^{q / p}\left(|a|^{p}+\beta_{p}\right)^{q / p} \\
& \leq(p+1)^{q / p}\left(|a|^{q}+\beta_{p}^{q / p}\right) .
\end{aligned}
$$

If $0<q \leq 1<p$, then we use Minkowski's inequality in the right-hand side of (2.3) to obtain 


$$
\begin{aligned}
\left.(q+1)|| a\right|^{q}-\beta_{q} \mid & \leq(p+1)^{q / p}\left(|a|+\beta_{p}^{1 / p}\right)^{q} \\
& \leq(p+1)^{q / p}\left(|a|^{q}+\beta_{p^{q} / p}^{q / p}\right) .
\end{aligned}
$$

Thus we get (2.2).

If $\hat{\beta}_{1}<\infty$ for $\mu$, denote the mean by $m$ and the central absolute moment of order $p$ by $\gamma_{p}$,

$$
\gamma_{p}=\int|x-m|^{p} \mu(d x) .
$$

THEOREM 2.2. Let $\mu$ be unimodal with a mode a. Let $p>1$. Then

$$
\left((q+1)^{1 / q}-(p+1)^{1 / p}\right)|a| \leq(q+1)^{1 / q} \beta_{q}^{1 / q}+(p+1)^{1 / p} \beta_{p}^{1 / p}
$$

for $1 \leq q<p$, and

$$
\begin{aligned}
& \left(2-(p+1)^{1 / p}\right)|a-m| \leq(p+1)^{1 / p} \gamma_{p}^{1 / p}, \\
& \left(2-(p+1)^{1 / p}\right)|a| \leq 2|m|+(p+1)^{1 / p} 3_{j}^{1 / p} .
\end{aligned}
$$

Proof. For $1 \leq q<p$, we apply Minkowski's inequality to the both sides of (2.4). Then we have

$$
(q+1)^{1 / 4}\left(|a|-\beta_{q}^{1 / q}\right) \leq(p+1)^{1 / p}\left(|a|+\beta_{p}^{1 / p}\right),
$$

which is identical with (2.4). In order to get (2.5), we let $q=1$ in (2.3), and then

$$
2 E|X-a| \leq(p+1)^{1 / p}\left(|m-a|+\gamma_{p}^{1 / t^{\prime}}\right) .
$$

Since $|m-a| \leq E|X-a|$, we have (2.5). The bound (2.6) follows from (2.5), because $\gamma_{p}^{1 / p} \leq|m|+\beta_{p}^{1 / p}$. The proof is complete.

THEOREM 2.3. For each $p>0$, there is a constant $A_{p}$ such that, if ": is unimodal with a mode a, then

$$
|a| \leq A_{p} \beta_{p}^{1 / p} .
$$

If $A_{p}$ is the best such constant, then $A_{p}$ is non-increasing in $p$ and

$$
\begin{array}{ll}
A_{p} \leq \frac{2+(p+1)^{1 / p}}{2-(p+1)^{1 / p}} & \text { for } p>1, \\
A_{p} \leq \inf _{0<q \leq 1} A_{p, q} & \text { for } 1<p<2, \\
A_{p} \leq \inf _{0<q<p} A_{p, q} & \text { for } 0<p \leq 1,
\end{array}
$$

where 


$$
A_{p .4}=\left(\begin{array}{l}
q+1+(p+1)^{q / p} \\
q+1-(p+1)^{q / p}
\end{array}\right)^{1 / q} \quad \text { for } 0<q \leq 1, q<p
$$

Proof. Use the moment inequality $\beta_{q}^{1^{\prime} q} \leq \beta_{p}^{1 / p}$ for $0<q<p$ in (2.2) or (2.4). Then we get

$$
\mid a_{i} \leq A_{3, q} p_{p}^{1 / p} \quad \text { for } 0<q<p .
$$

where $A_{p, q}$ is (2.11) or

$$
A_{i, q}=\frac{(q+1)^{1 / q}+(p+1)^{1 / p}}{(q+1)^{1 / q}-(p+1)^{1 / p}} \quad \text { for } 1 \leq q<p .
$$

This shows existence of $A_{p}$ satisfying (2.7). Let $A_{p}$ be the best such constant. The bounds (2.8)-(2.10) are immediate since $A_{p} \leq A_{p, q}$. Obviously $A_{p} \geq A_{p^{\prime}}$, for $p<p^{\prime}$.

Remark. For $p>1$, the $A_{p, q}$ of (2.13) is increasing in $q \in[1, p)$, since $(q+1)^{1 / q}$ is decreasing in $q$. Hence $\min _{1 \leq q<p} A_{p, q}=A_{p, 1}$. For $p \geq 2$, we have $\min _{0<q \leq 1} A_{p, q}=A_{p, 1}$ because $A_{p, q}$ is decreasing in $q \in(0,1]$. In fact, fix $p \geq 2$ and let $f(q)=q+1+(p+1)^{q / p}$ and $g(q)=q+1-(p+1)^{q / p}$ for $0<q \leq 1$. Then

$$
(d / d q) \log A_{g, ?}=-q^{-2} \log (f(q) / g(q))+q^{-1}\left(f^{\prime}(q) / f(q)-g^{\prime}(q) / g(q)\right)
$$

and we have

$$
\begin{aligned}
& g^{\prime}(q)=1-(p+1)^{q / p} p^{-1} \log (p+1) \geq g^{\prime}(1) \geq 1-3^{1 / 2} 2^{-1} \log 3>0 \\
& f(q) / g(q) \geq 1+2 / g(q) \geq 1+2 / g(1) \geq 3 \\
& f^{\prime}(q) / f(q) \leq 2^{-1} f^{\prime}(1) \leq 2^{-1}\left(1+3^{1 / 2} 2^{-1} \log 3\right)<1
\end{aligned}
$$

Hence $(d / d q) \log A_{\eta, q}<-q^{-2} \log 3+q^{-1}<0$.

\section{$\S 3$. Processes with homogeneous independent increments}

Let $X_{t}, t \geq 0$, be a real-valued process with homogeneous independent increments with $X_{0}=0$. We give estimates of its absolute moments. Let $\beta_{i}=E\left|X_{1}\right|^{p}$.

Theorem 3.1. Let $0<p \leq 1$. Suppose that $E\left|X_{t}\right|^{p}<\infty$. Then, for $0<q<p$,

$$
E: X_{b} \cdot \leq B_{q}\left(2 q^{-1}+e(p-q)^{-1}\right)\left(2^{2-p} \beta_{p} t\right)^{q / p} \quad \text { for } t \geq 1,
$$

where 


$$
B_{q}=2 \pi^{-1} \Gamma(q+1) \sin \left(2^{-1} q \pi\right) .
$$

Moreover, if $p \neq 1$, then, for $0<q \leq p$,

$$
E\left|X_{t}\right|^{q}=o\left(t^{q / p}\right) \quad \text { as } t \rightarrow \infty .
$$

Remark. For any $p>0$, the condition $E\left|X_{t}\right|^{p}<\infty$ for some $t>0$ implies $E \mid X_{t}^{i p}<\infty$ for all $t>0$, because this is equivalent to the condition $\int_{n>1} x_{i}^{p} \nu(d x)<\infty$ for the Lévy measure $\nu$ of $X_{t}([7],[8])$.

Proof of Theorem 3.1. Let $E \exp \left(i z X_{t}\right)=\varphi_{t}(z)=e^{-z}$, the characteristic function of $X_{t}$. Define $h_{t, p}(z)$ by

$$
\varphi_{t}(z)=1-|z|^{p} h_{t, p}(z) .
$$

Then, $h_{t, p}(z)$ is bounded in $z$. By Theorems 2.1 and 4.1 of Hsu [5], we have

$$
\begin{array}{ll}
\int_{-1}^{1}|z|^{-1}\left|h_{t, p}(z)\right| d z<\infty & \text { if } p<1, \\
\int_{-1}^{1}|z|^{-1} \mid \operatorname{Re} h_{t, p}(z) d z<\infty & \text { if } p=1,
\end{array}
$$

and

$$
E\left|X_{t}\right|^{p}=B_{p} \int_{0}^{\infty} z^{-1} \operatorname{Re} h_{t, p}(z) d z
$$

An explicit bound of $h_{t, p}(z)$ is known:

$$
\left|h_{t, p}(z)\right| \leq 2^{1-p} E\left|X_{t}\right|^{p} .
$$

Indeed,

$$
\begin{aligned}
\varphi_{t}(z)-1 \mid & \leq \int\left|e^{i z x}-1\right| \mu_{t}(d x)=2 \int\left|\sin 2^{-1} z x\right| \mu_{t}(d x) \\
& \leq\left. 2 \int\left|\sin 2^{-1} z x^{p} \mu_{t}(d x) \leq 2^{1-p}\right| z\right|^{p} \int x{ }^{n}, \cdot(d x),
\end{aligned}
$$

where $\because \because t$ is the distribution of $X_{t}$. Since

$$
|\log (1+w)|=\left|\int_{1}^{1+w} v^{-1} d v \leq 2\right| w \mid
$$

for any complex number $w$ satisfying $|w| \leq 2^{-1}$, we have

$$
\begin{aligned}
|y(z)| & =\left|\log \left(1-|\boldsymbol{z}|^{p} h_{1, p}(\boldsymbol{z})\right)\right| \leq 2|\boldsymbol{z}|^{p}\left|h_{1, p}(z)\right| \\
& \leq 2^{2-p} \beta_{p}|\boldsymbol{z}|^{p}=c^{-p}|\boldsymbol{z}|^{p}
\end{aligned}
$$


if $|z| \leq c$, where $c=\left(2^{2-p} \beta_{p}\right)^{-1 / p}$. Define $g_{p}(z)$ by $\psi(z)=|z|^{p} g_{p}(z)$. Now let $0<q<p$. Formula (3.7) with $p$ replaced by $q$ yields

$$
\begin{aligned}
E \mid X_{t}{ }^{\natural} & =B_{q} \int_{0}^{\infty} z^{-1} \operatorname{Re} h_{t, q}(z) d z \leq B_{q} \int_{0}^{\infty} z^{-q-1}\left|1-e^{t \psi(z)}\right| d z \\
& =B_{q} \int_{0}^{\infty} z^{-q-1}\left|1-\exp \left(t z^{p} g_{p}(z)\right)\right| d z=B_{q} t^{q / p}(I+J),
\end{aligned}
$$

where $I$ and $J$ are the integrals of $z^{-q-1}\left|1-\exp \left(z^{p} g_{p}\left(t^{-1 / p} z\right)\right)\right|$ over the intervals $(0, c)$ and $(c, \infty)$, respectively. Since $\left|e^{t \psi(z)}\right| \leq 1$, we have

$$
J \leq 2 \int_{c}^{\infty} z^{-q-1} d z=2 q^{-1} c^{-q} .
$$

Since

$$
\left|1-e^{w}\right|=\left|\int_{0}^{u} e^{v} d v\right| \leq|w| e^{i w \mid}
$$

for any complex $w$, we have

$$
I \leq \int_{0}^{r} z^{p-q-1}\left|g_{p}\left(t^{-1 / p} z\right)\right| \exp \left|z^{p} g_{p}\left(t^{-1 / p} z\right)\right| d z .
$$

It follows from (3.9) that

$$
I \leq e c^{-p} \int_{0}^{c} z^{p-q-1} d z=e(p-q)^{-1} c^{-q} .
$$

Thus we obtain (3.1).

Next we show (3.3). Let $0<q \leq p<1$. It follows from (3.5) that

$$
\int_{-1}^{1}|z|^{-1}\left|g_{p}(z)\right| d z<\infty
$$

By the calculation above, we have

$$
E\left|X_{t}\right|^{q} \leq B_{q} t^{q / p}(I(u)+J(u))
$$

for any $u>0$, where $I(u)$ and $J(u)$ are the integrals of $z^{-q-1} 1_{1}$ $\exp \left(z^{p} g_{p}\left(t^{-1 / p} z\right)\right)$ over intervals $(0, u)$ and $(u, \infty)$, respectively. For any given $\varepsilon>0$, we can find $u$ such that

$$
J(u) \leq 2 \int_{u}^{\infty} z^{-q-1} d z<\varepsilon
$$

Since $g_{p}(z)$ is locally bounded, there is a constant $K$ (depending on $u$ ) such that 


$$
\left|1-\exp \left(z^{p} g_{p}\left(t^{-1 / p} z\right)\right)\right| \leq K z^{p}\left|g_{p}\left(t^{-1 / p} z\right)\right|
$$

for $0 \leq z \leq u$ and $t \geq 1$. Hence, for $t \geq 1$,

$$
\begin{aligned}
I(u) & \leq K \int_{0}^{u} z^{p-q-1}\left|g_{p}\left(t^{-1 / p} z\right)\right| d z \\
& \leq K c^{p-q} \int_{0}^{u} z^{-1}\left|g_{p}\left(t^{-1 / p} z\right)\right| d z \leq K c^{p-q} \int_{0}^{u t-1 / p} z^{-1}\left|g_{p}(z)\right| d z,
\end{aligned}
$$

which tends to zero as $t \rightarrow \infty$ by virtue of (3.10). Thus (3.3) follows. The proof is complete.

Theorem 3.2. Let $1<p \leq 2$. Assume that $E\left|X_{t}\right|^{p}<\infty$ and $E X_{t}=0$. Then, for $1 \leq q<p$,

$$
E\left|X_{t}\right|^{q} \leq B_{q}\left(2 q^{-1}+e(p-q)^{-1}\right)\left(2^{3-p} p^{-1} \beta_{p} t\right)^{4 / p} \quad \text { for } t \geq 1
$$

where $B_{q}$ is given by (3.2). Moreover, if $p \neq 2$, then, for $1 \leq q \leq p$, we have

$$
E\left|X_{t}\right|^{4}=o\left(t^{q / p}\right) \quad \text { as } t \rightarrow \infty .
$$

Proof. Define $h_{t, p}(z)$ again by (3.4). By Theorem 2.1 of Hsu [5], (3.5) and (3.7) are true also for $1<p<2$. An explicit bound of $h_{t, p}(z)$ is

$$
\left|h_{t, p}(z)\right| \leq 2^{2-p} p^{-1} E\left|X_{t}\right|^{p}
$$

in place of (3.8), because

$$
\varphi_{t}(z)-1-\varphi_{t}^{\prime}(0) z=\int_{0}^{z}\left(\varphi_{t}^{\prime}(u)-\varphi_{t}^{\prime}(0)\right) d u
$$

and

$$
\begin{aligned}
\left|\varphi_{t}^{\prime}(z)-\varphi_{t}^{\prime}(0)\right| & \leq \int|x|\left|e^{i z . x}-1\right| \mu_{t}(d x)=2 \int|x|\left|\sin 2^{-1} z x\right| \mu_{t}(d x) \\
& \leq 2 \int|x|\left|\sin 2^{-1} z x\right|^{p-1} \mu_{t}(d x) \leq 2^{2-p}|z|^{p-1} \int|x|^{p} \mu_{t}(d x) .
\end{aligned}
$$

Letting $c=\left(2^{3-p} p^{-1} \beta_{p}\right)^{-1 / p}$, we have

$$
|\psi(z)| \leq c^{-p}|z|^{p} \quad \text { if }|z| \leq c .
$$

Now, if $1 \leq q<p$, then, starting with (3.7) for $E\left|X_{t}\right|^{q}$, we can proceed along the same line as the proof of (3.1) and obtain (3.11). If $1 \leq q \leq p$ and $1<p<2$, then the proof of (3.12) is wholly similar to that of (3.3).

From now on we assume that the distribution of $X_{t}$ is unimodal for every $t$. For example, if $X_{t}$ has distribution of class $L$, then it is unimodal, 
which is proved by Yamazato [13]. Let $a(t)$ be a mode of $X_{t}$. Let $m=$ $E X_{1}$ (if it exists) and $\gamma_{p}=E\left|X_{1}-m\right|^{p}$. To see asymptotic behavior of $a(t)$ as $t \rightarrow \infty$, we use the following lemma.

LEMma 3.1. Let $\left\{\mu_{n}\right\}$ be a sequence of probability measures that converges weakly to $\mu$. Suppose that, for each $n, \mu_{n}$ is unimodal with a mode $a_{n}, \mu$ is unimodal, and the mode $a$ of $\mu$ is unique. Then, $a_{n} \rightarrow a$.

This is obvious from the proof of Theorem 4 of Gnedendo-Kolmogorov [3], Section 32.

Theorem 3.3. Let $0<p \leq 1$ and assume that $E\left|X_{t}\right|^{p}<\infty$. Then

$$
|a(t)| \leq A_{q} B_{q}^{1 / q}\left(2 q^{-1}+e(p-q)^{-1}\right)^{1 / q}\left(2^{2-p} \beta_{p} t\right)^{1 / p} \quad \text { for } t \geq 1,
$$

where $q$ is an arbitrary number satisfying $0<q<p$. If $p \neq 1$, then

$$
a(t)=o\left(t^{1 / p}\right) \quad \text { as } t \rightarrow \infty .
$$

If $p=1$, then

$$
a(t)=m t+o(t) \quad \text { as } t \rightarrow \infty .
$$

Proof. The bound (3.14) is a conclusion of Theorems 2.3 and 3.1. In order to prove (3.15) for $p \neq 1$, choose $0<q \leq p$. Then (3.3) of Theorem 3.1 says that $E\left|t^{-1 / p} X_{t}\right|^{q} \rightarrow 0$ as $t \rightarrow \infty$. It follows that $t^{-1 / p} X_{t}$ tends to 0 in distribution. Hence we get (3.15) from Lemma 3.1. If $p=1$, then $t^{-1}\left(X_{t}-m t\right)$ tends to 0 in distribution by the law of large numbers. Thus we have (3.16) by Lemma 3.1. The proof is complete.

Theorem 3.4. Let $1<p \leq 2$ and let $E\left|X_{t}\right|^{p}<\infty$. Then

$$
a(t)=m t+o\left(t^{1 / p}\right) \quad \text { as } t \rightarrow \infty
$$

and

$$
|a(t)-m t| \leq C_{q} B_{q}^{1 / q}\left(2 q^{-1}+e(p-q)^{-1}\right)^{1 / q}\left(2^{3-p} p^{-1} \gamma_{p} t\right)^{1 / p} \quad \text { for } t \geq 1,
$$

where $q$ is an arbitrary number satisfying $1<q<p$ and

$$
C_{q}=\frac{(q+1)^{1 / q}}{2-(q+1)^{1 / q}}
$$

Proof. Let $Y_{t}=X_{t}-m t$. Then $Y_{t}$ is a process with homogeneous independent increments with mean 0 and $E\left|Y_{t}\right|^{p}<\infty$. It is unimodal with a mode $a(t)-m t$. Hence we get (3.18), combining Theorems 2.2 and 3.2. 
Let $p \neq 2$. By Theorem 3.2, we have $E\left|t^{-1 / p} Y_{t}\right|^{q} \rightarrow 0$ as $t \rightarrow \infty$ for $1 \leq q \leq p$. Thus $t^{-1 / p} Y_{t}$ tends to 0 in distribution and (3.17) follows from Lemma 3.1. In case $p=2$, the central limit theorem implies convergence of the distribution of $t^{-1 / 2} Y_{t}$ to a Gaussian distribution with mean 0 and, hence, $t^{-1 / 2}(a(t)-m t)$ tends to 0 by Lemma 3.1. This completes the proof.

Remark 1. In the proof we do not get any information on speed of convergence in the asymptotic behavior (3.15), (3.16), and (3.17) of the mode $a(t)$, because Lemma 3.1 does not tell anything about the speed. However, we can give an alternative proof to (3.15) for $0<p<1$ and to (3.17) for $1<p<2$ without resort to Lemma 3.1, combining the bounds (3.3) and (3.12) of absolute moments with the bounds (2.5) and (2.7) of the modes. So, in case $0<p<1$ or $1<p<2$, we can estimate speed of convergence in (3.15) or (3.17), if estimate of speed of convergence in (3.3) or (3.12) is given. In order to do this, estimate of the convergence

$$
\int_{-u}^{u}|z|^{-1}\left|h_{1, p}(z)\right| d z \rightarrow 0 \quad(u \rightarrow 0)
$$

is essential, as is seen from examination of the proof of (3.3) and (3.12). Concerning (3.20) we note

$$
\begin{gathered}
\int_{-u}^{u}|z|^{-1}\left|h_{1, p}(z)\right| d z=\int_{-u}^{u}|z|^{-p-1}\left|\varphi_{1}(z)-1\right| d z \\
\quad \leq \int_{-u}^{u}|z|^{-p-1} d z \int_{-\infty}^{\infty}\left|e^{i z x}-1\right| \mu_{1}(d x) \\
\quad=2 \int_{-\infty}^{\infty}|x|^{p} \mu_{1}(d x) \int_{0}^{u|x|}|z|^{-p-1}\left|e^{i z}-1\right| d z
\end{gathered}
$$

for $0<p<1$ and a similar relation

$$
\begin{aligned}
& \int_{-n}^{u}|z|^{-p-1}\left|\varphi_{1}(z)-1-\varphi_{1}^{\prime}(0) z\right| d z \\
& \quad \leq 2 \int_{-\infty}^{\infty}|x|^{p} \mu_{1}(d x) \int_{0}^{u|x|}|z|^{-p-1}\left|e^{i z}-1-i z\right| d z
\end{aligned}
$$

for $1<p<2$.

Remark 2. It is known that, if $E\left|X_{t}\right|^{p}<\infty$ for some $0<p<1$, then $t^{-1 / p} X_{t}$ tends to 0 almost surely as $t \rightarrow \infty$ ([2], [10]). This fact implies (3.15) by Lemma 3.1 . 


\section{$\S 4$. Stable processes with index 1}

Let $X_{t}$ be a stable process with index $0<\alpha<2$. A general form of its characteristic function is as follows:

$$
\begin{array}{ll}
\varphi_{t}(z)=\exp \left[t \lambda\left(i \gamma z-|z|^{\alpha}+i \sigma\left(\tan 2^{-1} \pi \alpha\right) z|z|^{\alpha-1}\right)\right] & (\alpha \neq 1), \\
\varphi_{t}(z)=\exp \left[t \lambda\left(i \gamma z-|z|-i \sigma 2 \pi^{-1} z \log |z|\right)\right] & (\alpha=1),
\end{array}
$$

where $\lambda, \gamma$, and $\sigma$ are real parameters, $\lambda>0,-1 \leq \sigma \leq 1$. The Lévy measure is supported on the positive half line if and only if $\sigma=1$. It is supported on the negative half line if and only if $\sigma=-1$. We assume that $\lambda=1$ and $\gamma=0$. It does not do harm to generality (consider $X_{t / \lambda}-t \gamma$ instead of $X_{t}$ ). It is a special case of Yamazato's result [13] that $X_{t}$ is unimodal for each $t$. Furthermore the mode of $X_{t}$ is unique for each $t$ (Sato-Yamazato [9]). We denote it by $a(t)$. When the index $\alpha$ is not one, Zolotarev [14] gives some information on $a(t)$. Thus he proves that

$$
\begin{array}{ll}
\operatorname{sgn} a(t)=\operatorname{sgn} \sigma & \text { if } 0<\alpha<1, \\
\operatorname{sgn} a(t)=-\operatorname{sgn} \sigma & \text { if } 1<\alpha<2,
\end{array}
$$

where $\operatorname{sgn} x=1,0,-1$ according as $x>0, x=0, x<0$, respectively. In case $\alpha=1$, however, to get information on $a(t)$ is more difficult. By numerical calculation he finds that $a(1)<0$ if $\alpha=1$ and $\sigma=k / 20, k=$ $1,2, \cdots, 20$ ([14] p. 172). But no proof is given to the assertion that $\operatorname{sgn} a(1)=-\operatorname{sgn} \sigma$ for $\alpha=1$.

We restrict our consideration to the case of index $\alpha=1$. Thus the characteristic function of $X_{t}$ is

$$
\varphi_{t}(z)=\exp \left[t\left(-|z|-i \sigma 2 \pi^{-1} z \log |z|\right)\right]
$$

Denote the mode of $X_{t}$ by $a_{\sigma}(t)$.

Proposition 4.1. (i) $a_{\sigma}(t)$ is a continuous function of two variables $(\sigma, t) . \quad a_{0}(t)=0, a_{\sigma}(0)=0, a_{-\sigma}(t)=-a_{\sigma}(t)$.

(ii) For any fixed $\sigma$,

$$
a_{\sigma}(t)=t a_{\sigma}(1)+2 \pi^{-1} \sigma t \log t .
$$

Let $0<\sigma \leq 1$. The derivative $a_{\sigma}^{\prime}(t)$ strictly increases from $-\infty$ to $+\infty$ as $t$ moves from 0 to $+\infty$. The mode $a_{\sigma}(t)$ strictly decreases from 0 to $a$ minimum negative value until an epoch $s_{\sigma}$ and then strictly increases to $+\infty$. There is a unique epoch $t_{\sigma}>0$ such that $a_{\sigma}\left(t_{\sigma}\right)=0$. 
(iii) $A s \quad \sigma \rightarrow 0$,

$$
a_{\sigma}(t)=\sigma\left(-K t+2 \pi^{-1} t \log t\right)+t O\left(\sigma^{3}\right),
$$

where $O\left(\sigma^{3}\right)$ does not depend on $t$ and

$$
K=\pi^{-1} \Gamma^{\prime}(3)>0 \text {. }
$$

(iv) As $\sigma$ decreases to 0 ,

$$
\begin{aligned}
& s_{\sigma}=\exp \left(-1+2^{-1} \pi K\right)+O\left(\sigma^{2}\right), \\
& t_{\sigma}=\exp \left(2^{-1} \pi K\right)+O\left(\sigma^{2}\right) .
\end{aligned}
$$

(v) There exists $T_{1}>0$ such that, if $0<t \leq T_{1}$ and $0<\sigma \leq 1$, then $a_{\sigma}(t)<0$.

(vi) There exists $T_{2}>0$ such that, if $t \geq T_{2}$ and $0<\sigma \leq 1$, then $a_{\sigma}(t)>0$.

(vii) An explicit bound of $a_{\sigma}(1)$ is

$$
\left|a_{\sigma}(1)\right| \leq A_{p} B_{p}^{1 / p}\left(2 p^{-1}+(1-p)^{-1}+4 \pi^{-2}(2-p)^{-3} \sigma^{2}\right)^{1 / p}
$$

for $0<p<1$, where $A_{p}$ and $B_{p}$ are of (2.7) and (3.2).

Proof. (i) Continuity of $a_{\sigma}(t)$ follows from Lemma 3.1 because $\varphi_{t}(z)$ is continuous in $(\sigma, t)$ for each $z$. If $\sigma=0$, then $X_{t}$ is symmetric and $a_{\sigma}(t)=0$. Since $X_{t}$ starts at the origin, $a_{\sigma}(0)=0$. The relation $a_{-\sigma}(t)=-$ $a_{\sigma}(t)$ is seen from (4.3).

(ii) The equality (4.4) is already observed by Zolotarev [14]. It is an easy consequence of the space-time relation

$$
\varphi_{t}(z)=\varphi_{1}(t z) \exp \left(i 2 \pi^{-1} \sigma z t \log t\right) .
$$

Since we have

$$
a_{\sigma}^{\prime}(t)=a_{\sigma}(1)+2 \pi^{-1} \sigma(1+\log t)
$$

from (4.4), the rest of the assertion is obvious.

(iii) It is enough to prove (4.5) for $t=1$. Let $f(x)$ be the density function of $X$. By the Fourier inversion we have

$$
f(x)=(2 \pi)^{-1} \int_{-\infty}^{\infty} \exp \left(-i x z-|z|-i 2 \pi^{-1} \sigma z \log |z|\right) d z .
$$

Write $a_{\sigma}(1)=a$. Since $a$ is the zero point of $f^{\prime}(x)$, we get

$$
\int_{-\infty}^{\infty} z \exp \left(-i a z-|z|-i 2 \pi^{-1} \sigma z \log |z|\right) d z=0 \text {. }
$$


Taking the imaginary part,

$$
\int_{0}^{\infty} z e^{-z} \sin \left(a z+2 \pi^{-1} \sigma z \log z\right) d z=0 .
$$

Using $|\sin x-x| \leq$ const $|x|^{3}$, we have

$$
\int_{0}^{\infty} z^{2} e^{-z}\left(a+2 \pi^{-1} \sigma \log z\right) d z+R=0
$$

where

$$
|R| \leq \text { const } \int_{0}^{\infty} z^{4} e^{-z}\left|a+2 \pi^{-1} \sigma \log z\right|^{3} d z \leq \text { const }\left(|a|^{3}+|\sigma|^{3}\right) .
$$

Therefore

$$
a+K \sigma=O\left(|a|^{3}\right)+O\left(|\sigma|^{3}\right)
$$

where

$$
K=\pi^{-1} \int_{0}^{\infty} z^{2} e^{-z} \log z d z=\pi^{-1} \Gamma^{\prime}(3)>0
$$

( $\Gamma^{\prime}$ is the derivative of the gamma function). Hence

$$
a\left(1+O\left(a^{2}\right)\right)=-K \sigma\left(1+O\left(\sigma^{2}\right)\right) .
$$

Since $a \rightarrow 0$ as $\sigma \rightarrow 0$ by (i), we have $a=-K \sigma(1+o(1))$ and hence

$$
a=-K \sigma\left(1+O\left(\sigma^{2}\right)\right) .
$$

(iv) is a consequence of (iii), as we have

$$
\begin{aligned}
& s_{\sigma}=\exp \left(-1-\pi(2 \sigma)^{-1} a_{\sigma}(1)\right), \\
& t_{\sigma}=\exp \left(-\pi(2 \sigma)^{-1} a_{\sigma}(1)\right)
\end{aligned}
$$

from (4.4) and (4.10).

(v) It follows from (iii) that there exists $\sigma_{1}>0$ such that $a_{\sigma}(1)<0$ for $0<\sigma \leq \sigma_{1}$. Hence $a_{\sigma}(t)<0$ for $0<\sigma \leq \sigma_{1}$ and $0<t \leq 1$. If $\sigma_{1} \leq \sigma \leq 1$ and $0<t \leq 1$, then

$$
a_{\sigma}(t) \leq t a_{\sigma}(1)+2 \pi^{-1} \sigma_{1} t \log t
$$

by (4.4). Since $a_{\sigma}(1)$ is bounded in $\sigma$ by continuity, it follows that $a_{\sigma}(t)<0$ for $\sigma_{1} \leq \sigma \leq 1$ if $t$ is small enough.

(vi) If $T>1$ is big enough, we see from (4.5) that $\sigma^{-1} a_{\sigma}(T)$ tends to a positive number as $\sigma \rightarrow 0$. Hence there is $\sigma_{2}>0$ such that $a_{\sigma}(T)>0$ for $0<\sigma \leq \sigma_{2}$. Hence $a_{\sigma}(t)>0$ for $0<\sigma \leq \sigma_{2}$ and $t \geq T$. Since 


$$
a_{\sigma}(t) \geq t a_{\sigma}(1)+2 \pi^{-1} \sigma_{2} t \log t
$$

for $\sigma_{2} \leq \sigma \leq 1$ and $t \geq 1$, we see, using boundedness of $a_{\sigma}(1)$ again, that $a_{\sigma}(t)>0$ for $\sigma_{2} \leq \sigma \leq 1$ if $t$ is sufficiently large.

(vii) Use (3.7). Then

$$
E\left|X_{1}\right|^{p}=B_{p} \int_{0}^{\infty} z^{-p-1}\left[1-e^{-z} \cos \left(2 \pi^{-1} \sigma z \log z\right)\right] d z=B_{p}(I+J),
$$

where $I$ and $J$ are the integrals over the intervals $(0,1)$ and $(1, \infty)$, respectively. We have

$$
\begin{aligned}
& I \leq \int_{0}^{1} z^{-p} d z+2 \pi^{-2} \sigma^{2} \int_{0}^{1} z^{1-p}(\log z)^{2} d z \\
& J \leq 2 \int_{1}^{\infty} z^{-\eta-1} d z
\end{aligned}
$$

using $1-e^{-z} \cos y \leq z+2^{-1} y^{2}$. Thus we obtain (4.9) from Theorem 2.3. The proof is complete.

Added in proof. The author has found the best constants $A_{p}$ and $D_{p}$ in the inequalities $|a| \leq A_{p} \beta_{p}^{1 / p} \quad(p>0)$ and $|a-m| \leq D_{p} \gamma_{p}^{1 / p} \quad(p \geq 1)$. The results are that $A_{p}$ is the unique zero point of $x^{p+1}-(p+1) x-p$ for $x>1$, and that $D_{p}=(p+1)^{1 / p}$. Proof will be published in Ann. Statist. Math. A under the title "Modes and moments of unimodal distributions".

\section{REFERENCES}

[1] Feller, W., An introduction to probability theory and its applications, Vol. II. Second edition, Wiley, New York, 1971.

[2] Fristedt, B. E., Sample function behavior of increasing processes with stationary, independent increments, Pacific J. Math., 21 (1967), 21-33.

[3] Gnedenko, B. V., Kolmogorov, A. N., Limit distributions for sums of independent random variables (English translation), Second edition, Addison-Wesley, Reading, 1968.

[ 4 ] Hall, P., On the limiting behaviour of the mode and median of a sum of independent random variables, Ann. Probab., 8 (1980), 419-430.

[5] Hsu, P. L., Absolute moments and characteristic function, Acta Chinese Math. Soc., 1 (1951), 259-280.

[6] Johnson, N. L., Rogers, C. A., The moment problem for unimodal distributions, Ann. Math. Statist., 22 (1951), 433-439.

[ 7 ] Kruglov, V. M., A note on infinitely divisible distributions, Theory Probab. Appl., 15 (1970), 319-324.

[8] Sato, K., A note on infinitely divisible distributions and their Lévy measures, Sci. Rep. Tokyo Kyoiku Daigaku, Sect. A, 12 (1973), 101-109. 
[ 9 ] Sato, K., Yamazato, M., On distribution functions of class L, Z. Wahrsch. Verw. Gebiete, 43 (1978), 273-308.

[10] Skorohod, A. V., Random processes with independent increments (in Russian), Nauka, Moscow, 1964.

[11] Vysochanskii, D. F. and Petunin, Yu. I., On a Gauss inequality for unimodal distributions. Theory Probab. Appl., 27 (1982), 359-361.

[12] Wolfe, S. J., Inequalities for modes of $L$ functions. Ann. Math. Statist., 42 (1971), 2126-2130.

[13] Yamazato, M., Unimodality of infinitely divisible distributions of class $L$, Ann. Probab., 6 (1978), 523-531.

[14] Zolotarev, V. M., One-dimensional stable distributions (in Russian), Nauka, Moscow, 1983.

Department of Mathematics

College of General Education

Nagoya University

Nagoya, Japan 